\title{
Reorientational dynamics of water confined in zeolites
}

\author{
Aoife C. Fogarty* François-Xavier Coudert $\dagger$ \\ Anne Boutin* and Damien Laage ${ }^{\ddagger *}$
}

November 27, 2013

\begin{abstract}
We present a detailed molecular dynamics study of water reorientation and hydrogen-bond dynamics in a strong confinement situation, within the narrow pores of an all-silica Linde type A (LTA) zeolite. Two water loadings of the zeolite are compared with the bulk case. Water dynamics is found to be retarded in this extreme hydrophobic confinement and the slowdown is more pronounced at higher water loading. We show that water reorientation proceeds mainly by large-amplitude angular jumps, whose mechanism is similar to that determined in the bulk. The slowdown upon hydrophobic confinement is found to arise predominantly from an excluded-volume effect affecting the large fraction of water molecules lying at the interface with the zeolite matrix, with an additional minor contribution coming from a structuring effect induced by the confinement.
\end{abstract}

Keywords: Water — Hydrogen-bond — Dynamics — Confinement — Zeolite

\section{Introduction}

Understanding the properties of water in confinement is of great importance in many contexts, including e.g. biochemistry where water can be found within narrow ion channels and protein cavities $^{[1,2]}$, geology where water can be confined in mineral inclusions and in clays ${ }^{[3]}$, but also in silica nanopores ${ }^{[4-6]}$, Nafion membranes used for fuel cells ${ }^{[4,7]}$, carbon nanotubes ${ }^{[8]}$, metal-organic frameworks ${ }^{[9,10]}$ and reverse micelles ${ }^{[11-15]}$.

Experiments and simulation studies have underlined the great impact of confinement on the structure, thermodynamics and dynamics of water ${ }^{[8,13,16]}$. The dynamics of confined water was measured to be retarded compared to the bulk situation, and several factors could be at the origin of this slowdown. However, their relative importance has not yet been clearly established. Possible factors include for example the chemical nature of the confining interface - hydrophilic or hydrophobic, polar or neutral ${ }^{[17]}$ - the shape of the interface, the size of the cavity and the density and structure of the confined liquid.

Here, we focus on a model system to investigate the impact of extreme confinement. We consider water confined within an all-silica narrow-pore zeolite. Zeolites are microporous crystalline materials which have a very wide industrial use for hydrocarbon separation and in detergents ${ }^{[18]}$. While most zeolites include sodium or aluminum ionic defects within their silica framework, we chose to study an all-silica zeolite ${ }^{[19,20]}$. This zeolite contains cages of $11 \AA$ in diameter which are hydrophobic due to the low polarity of the exposed oxygen atoms and to the absence of ionic or polar defects ${ }^{[21]}$, and in which water can be forced to enter under pressure ${ }^{[22]}$. Such a confining material is thus a good system to specifically investigate the effect of an extreme confinement on water dynamics, in the absence of specific interaction between water and the confining medium. We will focus here on water reorientation dynamics, which provides a good measure of the hydrogen $(\mathrm{H})$-bond network lability and dynamics, and which is accessible experimentally, for example via femtosecond infrared and NMR spectroscopies ${ }^{[23]}$.

\footnotetext{
*Department of Chemistry, UMR ENS-CNRS-UPMC 8640, rue Lhomond, F-75005 Paris, France

${ }^{\dagger}$ Chimie ParisTech and CNRS, 11 rue Pierre et Marie Curie, F-75005 Paris, France

‡corresponding author
} 
The outline of the remainder of this paper is as follows. In Section 2, we present the key features of the zeolite material, the two water loadings under consideration and the simulation procedure. Section 3 describes the structure of the water nanopools within the zeolite cages, and Section 4 compares the water reorientation dynamics within the zeolite with the bulk reference. Section 5 then uses the extended jump model ${ }^{[23]}$ recently developed by one of us to establish the molecular origin of the observed slowdown in water dynamics in strong hydrophobic confinement. Section 6 finally offers some concluding remarks and comparisons with other systems.

\section{Methodology}

\section{Zeolite Structure}

The structure of the zeolite is the cubic Linde type A, or LTA. This structure contains $\alpha$ cages of diameter $\simeq 13 \AA$, connected together in a cubic symmetry by 8 -membered oxygen ring windows of diameter $\simeq 6 \AA$, and smaller sodalite $\beta$ cages of diameter $\simeq 7 \AA$ (see Figure 1 ). This all-silica LTA zeolite was synthesised and characterised experimentally ${ }^{[19,20]}$. In our simulations, we employ a structure obtained by energy minimization at the Density Functional Theory level ${ }^{[24]}$. It is very similar to that determined in the experiments, containing a $\mathrm{Si}_{24} \mathrm{O}_{48}$ unit cell with a cubic $P m \overline{3} m$ symmetry and a lattice parameter of $11.9 \AA$. This unit cell contains one $\alpha$ supercage and one sodalite $\beta$ cage.

The pore volume of the $\alpha$ cages was calculated with an approach similar to that developed by Connolly ${ }^{[25]}$ and described in Refs. ${ }^{[26,27]}$. The solvent-accessible pore volume is delimited by the surface defined by rolling a probe sphere on the zeolite framework, defined as an assembly of hard spheres centered on the zeolite oxygens. The radii employed are $1.577 \AA$ for the water oxygen probe and $1.5 \AA$ for the zeolite oxygen sites, which correspond to half of the Lennard-Jones diameters of these respective sites within the force field employed here (see details below). The resulting accessible volume of one $\alpha$ supercage is $V_{\alpha}=754 \AA^{3}$, which would yield a $5.6 \AA$ radius for an ideal sphere. This value of the pore volume can then be used to determine the effective density of the confined liquid water. Two water loadings were simulated. In the first case (noted N=20 or high density), 20 water molecules per LTA unit cell were introduced, corresponding approximately to the saturation uptake in the liquid phase, as calculated by Grand Canonical Monte Carlo simulations ${ }^{[22]}$, and resulting in a 0.8 g.cm ${ }^{-3}$ mass density. This effective density is smaller than the bulk density in the same pressure and temperature conditions, consistent with earlier work on water density in other hydrophobic zeolites ${ }^{[26-29]}$. In the second case $(\mathrm{N}=15$ or low density), 15 water molecules per unit cell were considered, corresponding to a $0.6 \mathrm{~g} . \mathrm{cm}^{-3}$ mass density.

\section{Simulations}

Our simulations employ the rigid, non-polarisable SPC/E water model ${ }^{[30]}$ which has been shown to properly reproduce the dynamics of bulk water at room temperature. ${ }^{[31]}$ Due to the small size of the LTA unit cell, simulations are run on a $2 \times 2 \times 2$ supercell to reduce the influence of periodic boundary conditions. The positions of the zeolite framework atoms are fixed. Water-zeolite interactions are modelled using the force field described in Ref. ${ }^{[22]}$, which was shown to correctly reproduce the adsorption thermodynamics of water in all-silica zeolites ${ }^{[28]}$. Long-range electrostatic interactions are treated by Ewald summation and both repulsion-dispersion and electrostatic interaction energies between the rigid zeolite framework and water molecules are precomputed on a grid (with a grid mesh of $0.2 \AA$ ) and stored for use during the simulation.

After equilibration in the canonical ensemble at $300 \mathrm{~K}$, classical molecular dynamics are performed in the microcanonical ensemble with an average temperature of $304 \mathrm{~K}( \pm 1 \mathrm{~K})$. The propagation time step is $0.5 \mathrm{fs}$. All trajectories are run for more than $500 \mathrm{ps}$. The bulk water reference simulation with the same SPC/E water force field at $300 \mathrm{~K}$ is taken from a previous work ${ }^{[31]}$.

\section{Jump analysis}

The average jump mechanism, the jump angular amplitude and the jump time are determined using the same approach as described in detail in Ref. ${ }^{[31]}$. 


\section{Structure of water}

We first characterize the structural properties of water confined inside the LTA zeolite. At the lower water loading $(\mathrm{N}=15)$, all water molecules lie within the $\alpha$ cages or within the windows connecting them. At the higher water loading $(\mathrm{N}=20)$, one water molecule per unit cell is found within the sodalite $\beta$ cages while the remaining 19 water molecules are within the $\alpha$ cells. We therefore focus on water molecules within the $\alpha$ supercages.

We compute the radial distribution function between water oxygen sites. This is defined as

$$
g(r)=\frac{1}{\rho} \frac{\langle n(r)\rangle}{V(r)},
$$

where $\langle\ldots\rangle$ is an average over all water oxygen sites, $n(r)$ is the number of water oxygen atoms in a shell of width $\Delta r$ and radius $r$ around the oxygen center under consideration, $V(r)$ is the volume of that shell $V(r)=4 \pi r^{2} \Delta r$ and $\rho$ is the effective water oxygen number density within the $\alpha$ cage at the water loading under consideration, i.e. $\rho=N / V_{\alpha}$ where $V_{\alpha}=754 \AA^{3}$.

The resulting radial distribution functions are presented in Fig. 2a. The comparison with the radial distribution function in the bulk shows that the location of the first peak, corresponding to the H-bond length, has not changed significantly upon confinement. In contrast, the second peak, which reveals the position of the second shell, seems to shift closer to the central water oxygen upon confinement.

Compared with the bulk situation, the height of the first peak appears to be enhanced in confinement at high density, and unchanged in confinement at low density. However, the radial distribution function was computed with the standard definition used for bulk liquids. In the zeolite cages, a large fraction of the cell volume is not accessible to water, and this fraction changes with distance. We therefore calculate a new radial distribution function better suited for a confined fluid,

$$
g^{\prime}(r)=\frac{1}{\rho}\left\langle\frac{n(r)}{V^{\prime}(r)}\right\rangle .
$$

Here $V^{\prime}(r)$ is the volume of the shell at distance $r$ from the oxygen site which is accessible to water. This pore volume is calculated with the Connolly approach described in Sec. 2, using a stochastic sampling of the shell $V(r)$ to measure its overlap with the zeolite atoms and thus determine $V^{\prime}(r)$.

The resulting renormalized radial distribution functions are presented in Fig. 2b. The accessiblevolume correction shows that the first peak is only slightly enhanced in confinement compared to the bulk situation. The peak is more enhanced at low water loading, possibly because each water molecule forms fewer H-bonds than at higher loading and than in the bulk, thus reinforcing the existing H-bonds. The second peak location is now similar in confinement and in the bulk, which shows that the apparent shift seen in the standard radial distribution function Fig. 2a was an artefact due to the variation of the accessible volume with the distance. The third peak is dramatically enhanced in confinement, especially at higher water loading. This peak occurs at a distance which is approximately the separation between water molecules which are at the zeolite interface and diametrically opposed within the same $\alpha$ cage. At this distance, the probability to find a pair of water molecules remains finite while the water-accessible volume $V^{\prime}(r)$ becomes very small, leading to the observed enhancement of the radial distribution function.

\section{Water reorientational dynamics}

We now turn to the study of water reorientation and H-bond dynamics within the zeolite pores.

To measure the reorientation dynamics of water molecules, we compute the second-order reorientation time-autocorrelation function for a water $\mathrm{OH}$-bond vector $\mathbf{u}$, given by

$$
C_{2}(t)=\left\langle P_{2}[\mathbf{u}(0) \cdot \mathbf{u}(t)]\right\rangle,
$$

where $P_{2}$ is the second-order Legendre polynomial. For each system, we calculate $C_{2}(t)$ as a function of each $\mathrm{OH}$ group's H-bond acceptor at the time origin. This H-bond acceptor can be either another water oxygen $\left(\mathrm{O}_{W}\right)$ or in a small fraction of cases a zeolite oxygen $\left(\mathrm{O}_{Z}\right)$. The 
following geometric H-bond criteria are used: $R_{D A}<3.5 \AA, R_{A H}<2.5 \AA$, and $\theta_{H D A}<30^{\circ}$, where $D$ is the water oxygen $\mathrm{H}$-bond donor atom, $A$ the $\mathrm{H}$-bond acceptor oxygen atom and $H$ a hydrogen atom. In these zeolite systems, the overwhelming majority of the water $\mathrm{OH}$ groups lie within the first hydration shell of the zeolite, defined as containing all $\mathrm{OH}$ groups whose oxygen lies within $5 \AA$ of a zeolite oxygen. In both the high and low density systems, our results show that on average $96 \%$ of the water $\mathrm{OH}$ groups are in the first hydration shell, of which on average $4 \%$ point toward a zeolite oxygen atom and satisfy the H-bond geometric criteria.

The reorientation time-correlation functions are shown in Figure 3a, along with that of bulk water for comparison. In all cases, $C_{2}(t)$ displays an initial rapid sub-ps decline followed by a longertime component. We now discuss in detail what an examination of these two time components can tell us about water dynamics in confinement in a zeolite system. All values referred to in the following discussion are summarised in Table 1.

\section{Short time reorientation}

We first focus on the initial rapid ( $<200 \mathrm{fs}$ ) decay in $C_{2}(t)$. This sub-picosecond decay has been shown to be due to librational motions of the $\mathrm{OH}$ bond. ${ }^{[32]} \mathrm{In}$ an intact $\mathrm{H}$-bond, the $\mathrm{OH}$ group performs a librational or wobbling motion in a cone whose axis is the vector between the water oxygen $\mathrm{O}_{W}$ and the $\mathrm{H}$-bond acceptor oxygen (either a zeolite or water oxygen) $\mathrm{O}_{A}$, within the frame defined by the $\mathrm{O}_{W}-\mathrm{O}_{A}$ axis. This librational motion is the source of the initial rapid loss of correlation in the $C_{2}(t)$ function, and the size of the librational cone is a measure of H-bond strength, with cone angle increasing with decreasing H-bond strength. ${ }^{[32]}$ We have calculated the cone angles for all possible H-bonds in our systems, following the procedure detailed in Ref. ${ }^{[32]}$.

We first consider the $\mathrm{OH}$ groups initially $\mathrm{H}$-bonded to water oxygens $\mathrm{O}_{W}$. For these $\mathrm{OH}$ groups, the initial librational decay is approximately $20 \%$ of the initial correlation (Fig. 3a), which corresponds to a cone semiangle of $25-27^{\circ}$, a value very slightly below what is found for bulk water $\left(27^{\circ}\right)$. This suggests that the water-water H-bond is very slightly strengthened upon confinement. This is consistent with the small redshift of the $\mathrm{OH}$ stretch vibrational spectrum upon confinement that was computed for this zeolite ${ }^{[24]}$. The increase in water density, similarly, does not seem to have a strong impact on the cone semiangle.

Regarding the small fraction of water $\mathrm{OH}$ groups which point towards the zeolite and which engage in $\mathrm{H}$-bonds with the zeolite oxygen atoms $\mathrm{O}_{Z}$, the initial librational decay leads to a $\sim$ $50 \%$ loss of the orientational correlation (see Figure 3a), leading to cone semiangles of respectively $40^{\circ}$ and $35^{\circ}$ in the low and high water loadings. These values, which are markedly greater than for the $\mathrm{O}_{W} \mathrm{H} \cdots \mathrm{O}_{W} \mathrm{H}$-bonds, show that the $\mathrm{H}$-bond with the zeolite matrix is much weaker than with water. This is consistent with the large-amplitude librations already found for water next to sites whose polarity is low ${ }^{[3,34]}$. The polarity of the zeolite oxygen sites is so low that the water $\mathrm{OH}$ groups pointing toward the interface can almost be characterized as dangling $\mathrm{OH}$ bonds. These very weakly H-bonded groups appear as a small blue-shifted peak in the vibrational stretch spectrum ${ }^{[24,33]}$ and have been extensively characterized experimentally at water-organic solvent interfaces ${ }^{[35]}$.

\section{Long time reorientation}

We now turn to the long-time component in the decay of the reorientation time-correlation function $C_{2}(t)$ (Eq. 3). It is quantified for each initial H-bond acceptor in each system via the secondorder reorientation time $\tau_{\text {reor }}^{M D}$ extracted from a monoexponential fit of $C_{2}(t)$ in the 2-10 ps time interval. The most striking observation is the slowdown in water reorientational dynamics caused by confinement, with reorientation times of $7.3 \mathrm{ps}$ and $5.2 \mathrm{ps}$ for water initially H-bonded to $\mathrm{O}_{W}$ in the high and low density zeolite systems respectively, compared to $2.5 \mathrm{ps}$ in bulk water. Secondly, increasing water density in the zeolite slows down reorientational relaxation, with reorientation times of 7.3 ps for an initial H-bond to $\mathrm{O}_{W}$ and 5.5 ps for an initial H-bond to $\mathrm{O}_{Z}$ at high density, compared to $5.2 \mathrm{ps}$ and $4.8 \mathrm{ps}$ respectively at low density. Finally, the identity of the initial Hbond acceptor also influences reorientational dynamics. At a given density, a water $\mathrm{OH}$ group initially $\mathrm{H}$-bonded to $\mathrm{O}_{Z}$ reorients more quickly than an $\mathrm{OH}$ group initially $\mathrm{H}$-bonded to $\mathrm{O}_{W}$. The 
reorientation times are 5.5 ps vs 7.3 ps respectively in the high density system and 4.8 ps vs 5.2 ps respectively in the low density system.

In the next section, these observations will be explained using a theoretical model for water reorientation, the extended jump model, introduced by one of us. ${ }^{[23,36,37]}$ This model will be used to precisely identify which molecular factors cause the measured slowdown in water dynamics within zeolites.

\section{$5 \quad$ Extended jump model analysis}

It has been shown that the main contribution to water reorientation both in the bulk and next to a range of solutes ${ }^{[23,38]}$, as well as in confinement in silica nanopores, ${ }^{[6]}$ consists of large-amplitude angular jumps in which the reorienting $\mathrm{OH}$ group changes $\mathrm{H}$-bond acceptors. We first demonstrate that these jumps are present within the zeolite pores and recall the main features of the extended jump model which connects the jump features to the water reorientation time. We then use the model to establish the relative importance of the different factors affecting water dynamics in strong hydrophobic confinement.

\section{Characterization of angular jumps in zeolites}

Large amplitude angular jumps were recently suggested ${ }^{[39]}$ to occur in chabazite, a narrow-pore zeolite containing ionic defects. Here, we provide a confirmation and detailed characterization of these jumps.

We perform an a posteriori analysis of our MD trajectories, identifying each H-bond exchange event, i.e. each time a water hydroxyl group $\mathrm{O}^{*} \mathrm{H}^{*}$ that was initially $\mathrm{H}$-bonded to a water or zeolite oxygen $\mathrm{A}^{i}$ becomes H-bonded to a different water oxygen $A^{f}$. We define $\theta$ as the angle between the $\mathrm{O}^{*} \mathrm{H}^{*}$ bond and the bisector plane of $A^{i} \widehat{O^{*}} A^{f}$, and $\phi$ as the $A^{i} \widehat{O^{*}} A^{f}$ angle. We also define distances $R_{O * A^{i}}, R_{O * A^{f}}$ and $R_{A^{i} A^{f}}$. All parameters are illustrated in Figure 4 . For each H-bond exchange event, we calculate these geometric parameters as a function of time during the 1 ps directly preceding and following the event. We examine more than 5000 such events for an initial $\mathrm{O}_{W}$ acceptor and more than 1000 events for an initial $\mathrm{O}_{Z}$ acceptor. We define a common time origin for each trajectory as the moment when $\theta=0$. This allows us to calculate the average time evolution of each geometric parameter as a function of the type of initial H-bond acceptor in each system, presented in Figure 5.

We find a large-amplitude angular jump mechanism for reorientation in each case, as evidenced by the data presented in Figure 5. The details of the mechanism are qualitatively similar in each zeolite system and in bulk water. The key features are the departure of the initial H-bond acceptor, seen in the increase in $R_{O * A^{i}}$ after the transition state, the approach of the final acceptor to form a new $\mathrm{H}$-bond, seen in the decrease in $R_{O * A^{f}}$, and the abrupt change in $\theta$ when the OH group switches from one acceptor to the other. We now discuss the quantitative differences between the systems.

Firstly, comparing the bulk water trajectories to those in the zeolite systems, we see that in confinement the average value of $R_{O * A^{f}}$ before the H-bond exchange process is between 3.5 and $4.0 \AA$ versus $\sim 4.5 \AA$ in the bulk. Similarly, the final value of $R_{O * A i}$ after the exchange is smaller in confinement than in the bulk. The first hydration shell around a given water molecule peaks at $2.8 \AA$ in terms of oxygen-oxygen distance, while the second hydration shell lies between 4.4 and $4.6 \AA$ (see Figure $2 \mathrm{~b}$ ). While in the bulk the new H-bond partner $A^{f}$ comes on average from the second hydration shell ${ }^{[31]}$, in confinement the new partner in a significant proportion of jumps comes from the first hydration shell, leading to a lower average initial value of $R_{O * A^{f}}$. A similar argument holds for the destination of the initial H-bond partner $A^{i}$ and the lower final value of $R_{O * A i}$ in confinement.

Secondly, we see that $R_{A^{i} A^{f}}$ and $\phi$ are much larger for $\mathrm{O}_{Z} \rightarrow \mathrm{O}_{W}$ jumps than $\mathrm{O}_{W} \rightarrow \mathrm{O}_{W}$ jumps, with values of 3.75 to $5.5 \AA$ and 70 to $110^{\circ}$ versus 3 to $3.5 \AA$ and $\sim 60^{\circ}$ respectively. This is because a water $\mathrm{OH}$ group that initially points toward the zeolite must undergo a larger amplitude jump to find a new water H-bond acceptor which usually comes from behind it. A similar effect had been described for water at an apolar interface ${ }^{[33][34]}$. The jump amplitude is defined as the 
value of the angle $\phi$ between the initial $\mathrm{O}^{*} \mathrm{H}^{*} \cdots \mathrm{A}^{i}$ and final $\mathrm{O}^{*} \mathrm{H}^{*} \ldots \mathrm{A}^{f} \mathrm{H}$-bond axes in the jump transition-state geometry. Again, values are given in Table 1. When the water density within the pore increases, the jump amplitude for $\mathrm{O}_{Z} \rightarrow \mathrm{O}_{W}$ jumps decreases slightly because the new partner can be more frequently found within the interfacial layer. The amplitude of the $\mathrm{O}_{W} \rightarrow \mathrm{O}_{W}$ jump is little affected by confinement in the zeolite or by a change in water density.

In summary, while confinement causes quantitative changes in the details of the H-bond exchange process, particularly for $\mathrm{O}_{Z} \rightarrow \mathrm{O}_{W}$ jumps, the fundamental jump mechanism underlying water reorientation remains unchanged.

\section{Extended Jump Model}

Having demonstrated the existence of large-amplitude angular jumps in the water reorientation mechanism in zeolites, we then go on to apply the extended jump model (EJM) ${ }^{[23]}$ to connect these jumps to the measured water reorientation time in the zeolite systems.

Jumps are an activated process, and can usefully be treated as chemical reactions, i.e. a process whose rate constant can be determined by the free energy difference between its reactant and transition states. The jump kinetics can be followed via the cross time-correlation function (tcf) between stable states ${ }^{[31]} \mathrm{I}$ and $\mathrm{F}$ (in which the $\mathrm{OH}$ group forms a stable H-bond with the initial and final acceptors respectively). This jump tcf is given by ${ }^{[23]}$

$$
C_{\text {jump }}(t)=\left\langle n_{I}(0) n_{F}(t)\right\rangle,
$$

where $n_{I, F}=1$ if the $\mathrm{OH}$ group is in stable state I and F respectively, and $n_{I, F}=0$ otherwise. Absorbing boundary conditions are used in the product state, so that only the first jump performed by each $\mathrm{OH}$ group is considered. The jump time, $\tau^{\text {jump }}$, which is the inverse of the rate constant for the H-bond acceptor exchange, can then be found via a monoexponential fit of $1-C_{\text {jump }}(t)$ in the $0.5-10 \mathrm{ps}$ time interval. The amplitude of the jump angle $\Delta \theta$ has an average bulk water value of $68^{\circ},{ }^{[31]}$ and deviates from this value in the presence of a solute or in confinement.

A secondary and minor contribution to water reorientation arises from the tumbling of the molecular frame, i.e. the reorientation of an intact $\mathrm{H}$-bond between jumps. ${ }^{[31]}$ The time constant for this process, $\tau_{\text {reor }}^{\text {frame }}$, can be extracted from an exponential fit of the $C_{2}(t)$ reorientation correlation function of the $\mathrm{O}_{W}-\mathrm{H}$ vector between jumps, an approximation of the $\mathrm{O}_{W}-\mathrm{O}_{A}$ vector between jumps.

The jump and frame-tumbling contributions to reorientation can then be combined in the extended jump model to give a predicted second-order reorientation time, thus ${ }^{[31]}$

$$
\frac{1}{\tau_{\text {reor }}^{\text {EJM }}}=\frac{1}{\tau_{\text {reor }}^{\text {jump }}}+\frac{1}{\tau_{\text {reor }}^{\text {frame }}},
$$

where the jump contribution is given by ${ }^{[31]}$

$$
\tau_{\text {reor }}^{\text {jump }}=\tau^{\text {jump }} /[1-\sin (5 \Delta \theta / 2) /(5 \sin (\Delta \theta / 2)] .
$$

The EJM model has been shown to give results in agreement with both NMR and pump-probe infrared experiments on bulk water, as well as with reorientation times calculated directly from molecular dynamics simulations. ${ }^{[37]}$ It also holds true for water reorientation in confinement in zeolites, as we now show.

The values resulting from application of the EJM are given in Table 1. Comparison of extended jump reorientation times from the model $\left(\tau_{\text {reor }}^{E J M}\right)$ with reorientation times directly calculated from simulation $\left(\tau_{\text {reor }}^{M D}\right)$ shows that the model correctly reproduces reorientation times in each case. The extended jump model can therefore be used to analyze and understand reorientational dynamics in these systems. We now use this model to elucidate the molecular origins of the effects of confinement and changes in density and the initial H-bond acceptor on reorientational dynamics.

\section{Origin of slowdown}

Our principal observation was the slowdown in water reorientation upon confinement in zeolites. We first concentrate on the comparison between reorientation times for $\mathrm{OH}$ groups initially $\mathrm{H}$ bonded to water oxygen atoms, in either bulk water or the zeolite, since they represent the dominant population within the pores. 
Table 1 shows that the frame contribution slows down dramatically in confinement. However, a comparison of the jump and frame reorientation times shows that the frame is a minor contribution to the overall reorientation time and it is in fact the slowdown in the jump component which is the main cause of the overall reorientation retardation. Within the EJM, the jump reorientation time is only determined by the jump amplitude and by the jump time, and the values reported in Table 1 unambiguously show that the $\tau^{\text {reor }}$ retardation arises from a slowdown in the H-bond jump time $\tau^{\text {jump }}$.

We now analyze the origin of this slowdown in the jump time. The retardation factor compared with the bulk case is respectively 2.1 and 2.3 in the low and high zeolite water loading (see Table 1). A first cause of this slowdown comes from an excluded-volume effect for water $\mathrm{OH}$ groups lying at the interface with the zeolite wall. Our structural analysis has shown that they represent the overwhelming majority $(\simeq 96 \%)$ of the water molecules within the pores. Since part of the rate-limiting step in the $\mathrm{H}$-bond jump mechanism is the approach of a new H-bond partner, the presence of a solute or an interface hinders this approach and leads to an excluded-volume effect at the jump transition state which results in a slowdown in the jump rate constant. This was described in detail for water molecules next to small hydrophobic convex solutes ${ }^{[40]}$ and next to flat apolar interfaces ${ }^{[33,34]}$.

Starting from the simple anaytic prediction of the jump slowdown factor that was derived for water next to a spherical convex solute in Ref. ${ }^{[40]}$, it is straightforward to show that for water within a spherical concave pore, the slowdown factor can be approximated as

$$
\rho_{\text {jump }}=\left\{1 / \pi \arccos \left[\frac{r^{2}-2 r R+\left(R^{\ddagger}\right)^{2}-R^{\ddagger} d \cos (\Delta \theta)}{2 R^{\ddagger} \sin (\Delta \theta) \sqrt{(R-r)^{2}-(d / 2)^{2}}}\right]\right\}^{-1},
$$

where $R$ is the radius of the spherical cavity, $r$ is the distance between the interfacial hydration layer and the minimum approach position next to the pore, $R^{\ddagger}$ is the distance between oxygen atoms at the jump transition state, $\Delta \theta$ is the amplitude of the angular jump and $d$ is the average distance between two $\mathrm{H}$-bonded water oxygen atoms. With typical values for these parameters $\left(R^{\ddagger}=3.2 \AA, \Delta \theta=60^{\circ}, d=2.8 \AA\right.$ and $\left.r=0.8 \AA\right)$ and with the radius of the zeolite pores under consideration $R=5.6 \AA$, the predicted excluded-volume slowdown factor is $\rho_{\text {jump }} \simeq 1.9$. This value is very close to what was previously determined next to a flat apolar surface ${ }^{[33,34]}$.

An additional contribution to the overall slowdown in the jump time arises from the enhancement of the water structure induced by the confining interfaces. While the effect of a single interface disappears after a few hydration layers, the confinement within the narrow pores of the zeolite studied here causes a strong structuring effect. This can be seen in the radial distribution functions presented in Fig. $2 \mathrm{~b}$ which reveal an enhancement of the peaks and of the dips compared with the bulk situation. This increased local structure leads to a greater free energy cost for the elongation of the initial H-bond and for the approach of the new H-bond partner predominantly originating from the second shell, which were shown to bring a dominant contribution to the jump free energy barrier ${ }^{[31]}$. As shown in Ref. ${ }^{[31]}$, the jump free energy barrier can be approximated as

$$
\Delta G^{\ddagger} \simeq w\left(R_{O * A^{i}}^{\ddagger}\right)-w\left(R_{O * A^{i}}^{R}\right)+w\left(R_{O * A^{f}}^{\ddagger}\right)-w\left(R_{O * A^{f}}^{R}\right),
$$

where $\mathrm{O} *$ is the central rotating water oxygen, $A^{i}$ and $A^{f}$ are the initial and final $\mathrm{H}$-bond acceptors, $R^{\ddagger}$ is the distance at the jump transition state, and $R^{R}$ is the value in the reactants, i.e. the initial stable H-bond. $w(r)$ is the potential of mean force along the oxygen-oxygen distance (Fig. 6), computed from the radial distribution function

$$
w(r)=-k_{B} T \ln \left[g^{\prime}(r)\right] .
$$

Using the $R^{\ddagger}$ and $R^{R}$ values determined from the average jump mechanisms in Fig. 5, the slowdown induced by the confinement-induced structuring is $\rho=\left(\Delta G_{\text {zeolite }}^{\ddagger}-\Delta G_{\text {bulk }}^{\ddagger}\right) / k_{B} T \simeq 1.2 \pm 0.2$.

When combined together, these two slowdown factors lead to a satisfactory agreement with the directly computed retardation factor for the jump time (2.1 and 2.3 in the low and high density systems respectively). Our analysis has thus separated the contributions arising from an excludedvolume effect on interfacial water molecules and from a confinement-induced structuring effect. Although our estimates are not fully quantitative, our approach can already clearly show that the 
dominant effect arises from the steric excluded-volume effect due to the large fraction of water molecules lying at the interface, with an additional contribution from the structuring effect.

We further note that the faster reorientation dynamics measured at a given water density for the water $\mathrm{OH}$ groups which are initially pointing towards the zeolite oxygen atoms $\mathrm{O}_{Z}$ compared to those $\mathrm{H}$-bonded to water oxygens $\mathrm{O}_{W}$ can also be rationalized by the extended jump model approach. Table 1 shows that this shorter reorientation time is due to a shorter jump time for $\mathrm{H}$-bond exchange for $\mathrm{O}_{Z} \rightarrow \mathrm{O}_{W}$ jumps than for $\mathrm{O}_{W} \rightarrow \mathrm{O}_{W}$ jumps. This jump time in turn depends on the free energy cost to stretch the initial H-bond to the jump transition state ${ }^{[23]}$. As noted above from an examination of the librational cone size, an $\mathrm{O}_{W} \mathrm{H} \cdots \mathrm{O}_{Z} \mathrm{H}$-bond is weaker than an $\mathrm{O}_{W} \mathrm{H} \cdots \mathrm{O}_{W} \mathrm{H}$-bond, which facilitates the $\mathrm{H}$-bond jumps and thus the reorientation.

Finally, we observe water dynamics to depend on the density of water in the zeolite. Increasing water density slows down reorientational relaxation. For an $\mathrm{OH}$ group initially $\mathrm{H}$-bonded to a water oxygen, $\tau_{\text {reor }}$ is $5.2 \mathrm{ps}$ at low density and $7.3 \mathrm{ps}$ at high density, while the corresponding values for an $\mathrm{OH}$ groups initially $\mathrm{H}$-bonded to a zeolite oxygen are 4.8 and $5.5 \mathrm{ps}$. This slowdown is dominated by an increase in $\tau_{\text {reor }}^{\text {frame }}$, which is very sensitive to density. Frame tumbling is a diffusive motion, and is therefore retarded by the increasing viscosity which comes from increasing density. (We note that the frame tumbling time becomes infinite for $\mathrm{OH}$ groups $\mathrm{H}$-bonded to $\mathrm{O}_{Z}$ sites because the zeolite framework is fixed and this H-bond cannot tumble.)

\section{Conclusions}

We have shown that within the narrow, $11 \AA$-diameter hydrophobic pores of an all-silica zeolite, water reorientation occurs through large amplitude jumps. Water reorientation is retarded by a factor of 2-3 compared with the bulk situation. Most of this slowdown is due to an excluded-volume effect which affects the water molecules at the zeolite interface. Additional smaller contributions arise from the enhanced water structure induced by the confinement within the narrow zeolite pores and from the slower reorientation of intact H-bonds between successive H-bond jumps.

The present study has determined and analyzed the effect on water dynamics of a very strong confinement within a matrix which is hydrophobic and has no specific interaction with the water molecules. Other studies of wider nanopores ${ }^{[5,6]}$ had found a retardation factor of approximately 2 for the interfacial water molecules, which is consistent with our present results. At the interface with large polar nanopores ${ }^{[5,6]}$ and within reverse micelles of sizes similar to the zeolite cages studied here ${ }^{[11-15]}$, the presence of the strongly hydrophilic surfactant headgroups leads to a much greater slowdown of water dynamics. While the effect on water dynamics of hydrophilic defects within hydrophobic zeolites ${ }^{[21]}$ will deserve a further specific study, these results together with our present study thus already suggest that the presence of the interface and its chemical nature have a much greater impact on water dynamics than confinement.

\section{Aknowledgements}

DL thanks Guillaume Stirnemann (ENS) for fruitful discussions. 


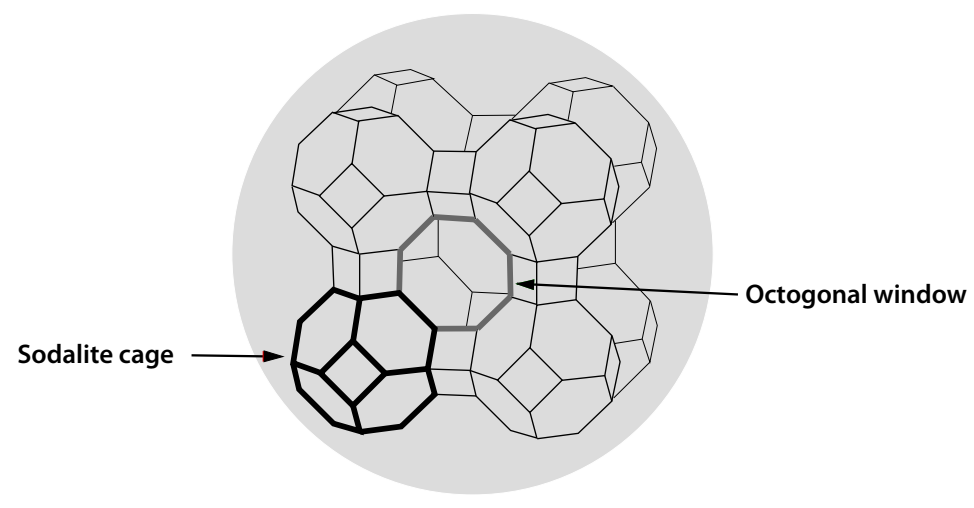

Figure 1: Schematic view of the LTA structure showing an $\alpha$ cage (centre) with its eight surrounding sodalite $\beta$ cages. $\alpha$ cages are connected together by octogonal windows.

a

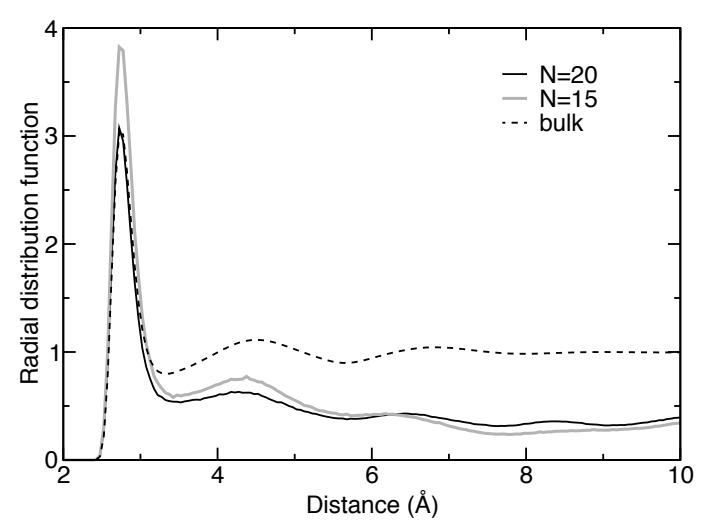

b

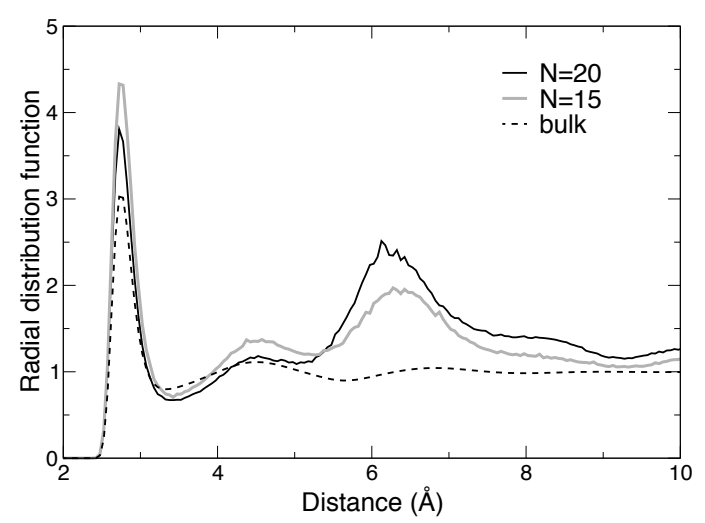

Figure 2: a) Standard radial distribution function (Eq. 1) between water oxygen atoms in bulk water and in the two zeolite systems. b) Same radial distribution functions corrected for accessible volume effects (Eq. 2). 
a

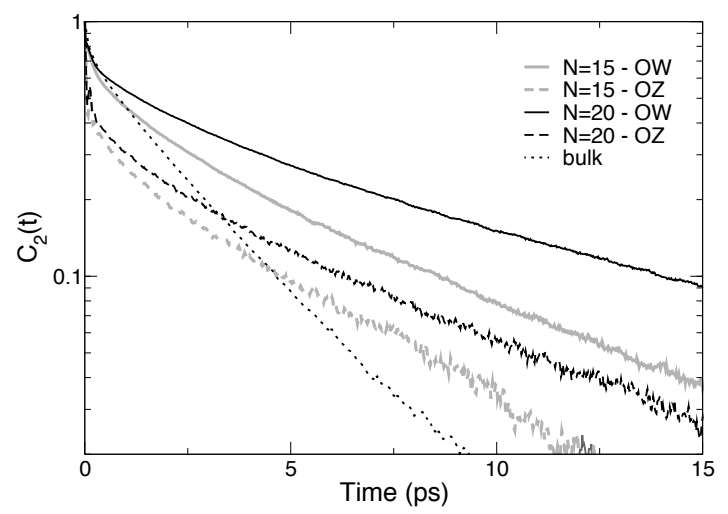

b

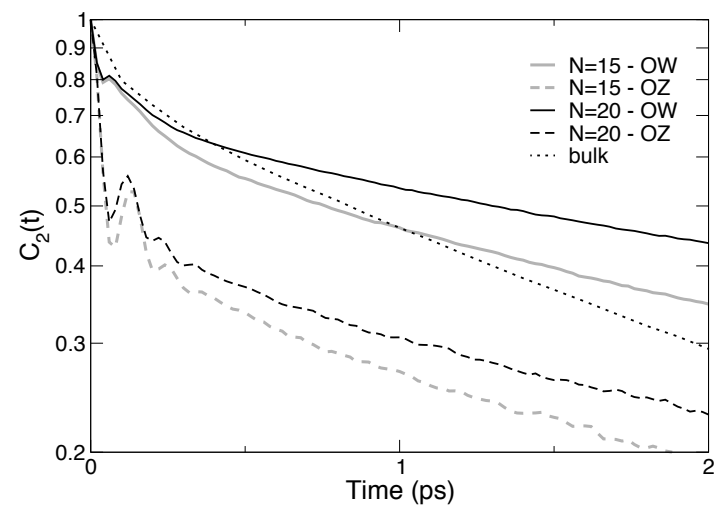

C

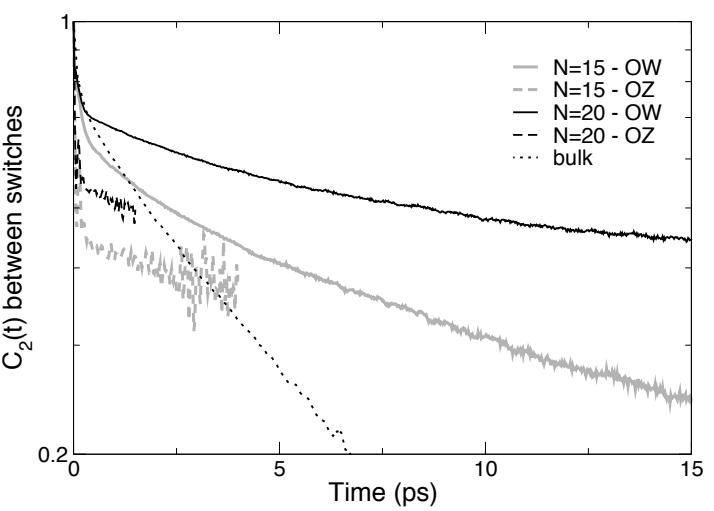

Figure 3: (a) Reorientation time-correlation functions $C_{2}(t)$ (Eq. 3) for water $\mathrm{OH}$ groups, in bulk water and in low and high density zeolite systems, as a function of the initial H-bond acceptor, which can be either another water oxygen $\left(\mathrm{O}_{W}\right)$ or a zeolite oxygen $\left(\mathrm{O}_{Z}\right)$. (b) Idem focussing on the initial decay. (c) Frame reorientation time-correlation functions, i.e. reorientation timecorrelation functions $C_{2}(t)$ for $\mathrm{OH}$ bonds between successive H-bond acceptor exchanges, for the same five subsets of $\mathrm{OH}$ groups. 


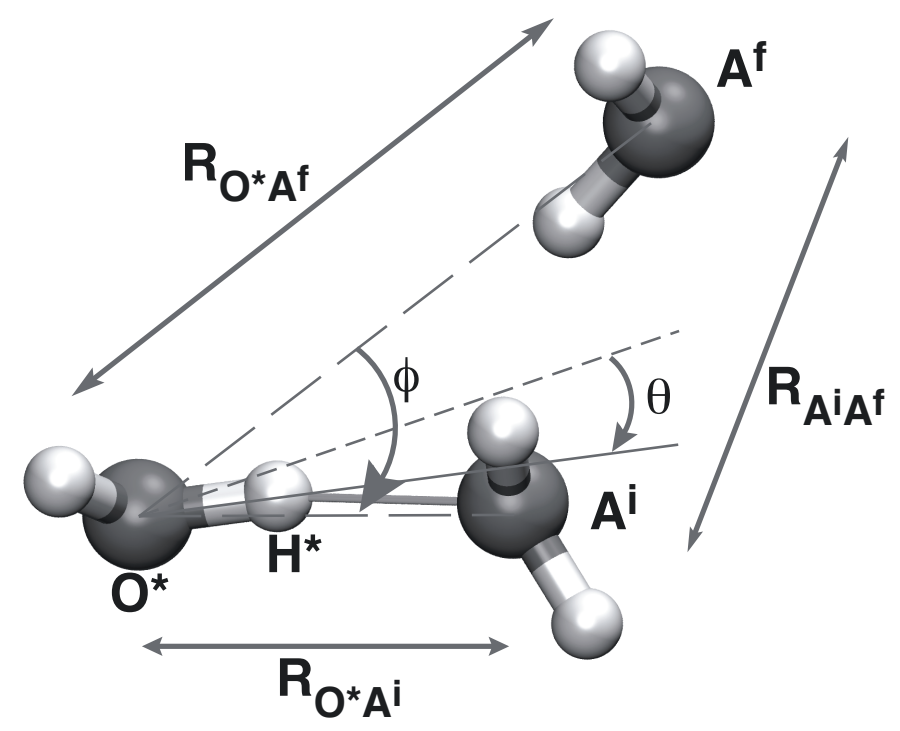

Figure 4: Definition of the different geometric parameters used to follow the H-bond exchange event, as described in the text. 
Ow to Ow jump
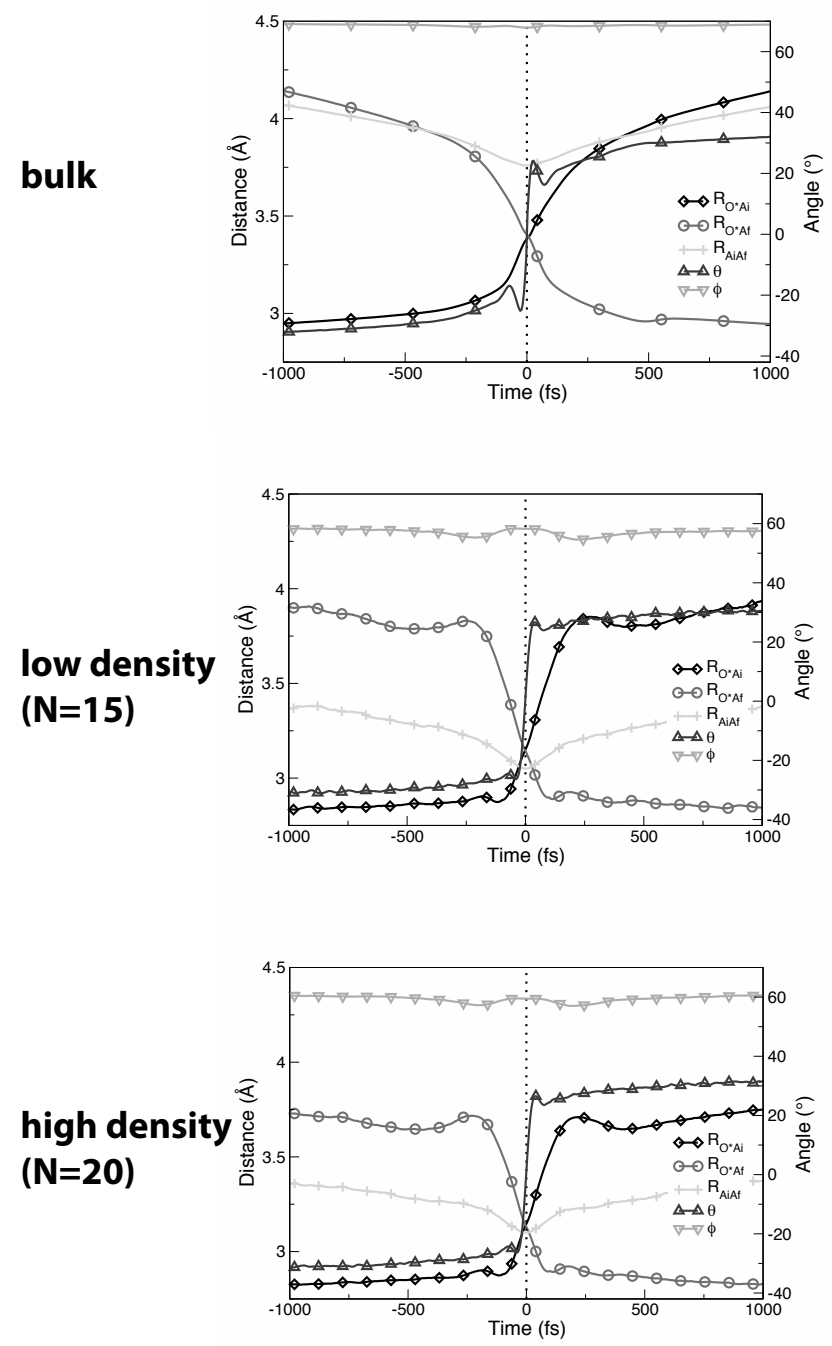

Oz to Ow jump
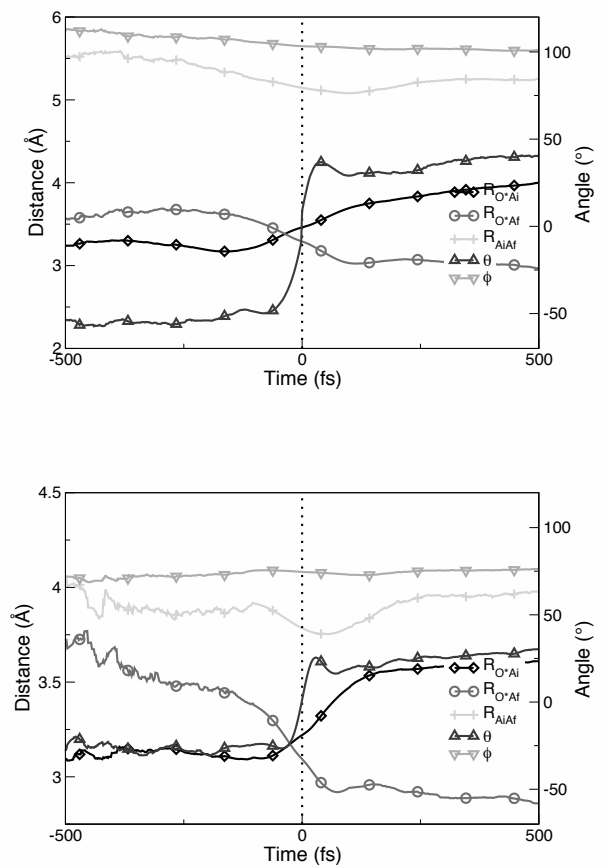

Figure 5: Average H-bond exchange trajectories for the three systems studied (bulk water and low and high density zeolite systems) and the two possible initial H-bond acceptors, $\mathrm{O}_{W}$ (water oxygen) and $\mathrm{O}_{Z}$ (zeolite oxygen). Geometric coordinates are as defined in the text and in Fig. 4. The time origin is defined by $\theta=0$. 


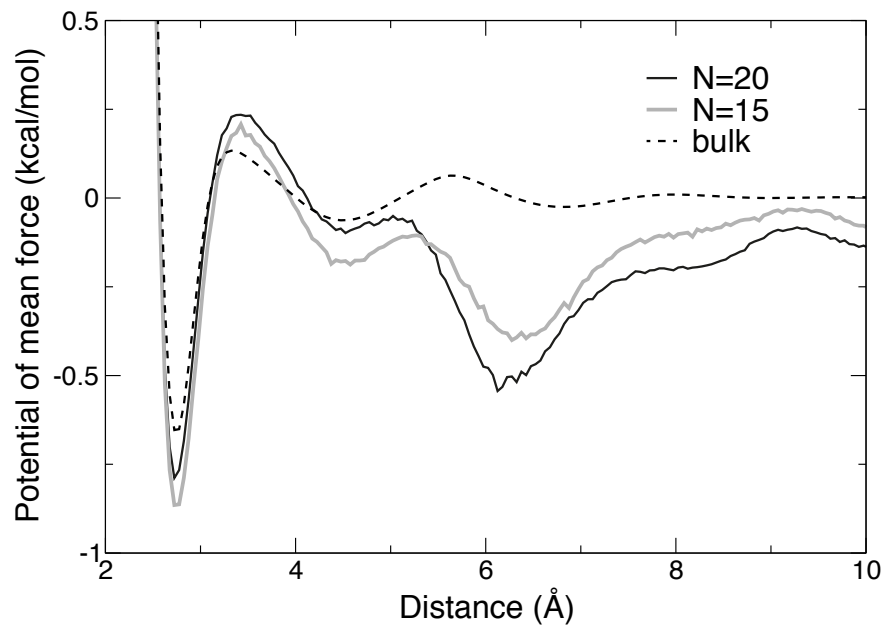

Figure 6: Potential of mean force as a function of water oxygen-water oxygen distance calculated using the $g^{\prime}(r)$ radial distribution functions modified to take into account the volume accessible to the confined water (Eq. 2). 


\begin{tabular}{c|ccccc} 
System & \multicolumn{2}{|c}{$\begin{array}{c}\text { Low density water in zeolite } \\
0.6 \text { g.cm }^{-3}\end{array}$} & $\begin{array}{c}\text { High density water in zeolite } \\
0.8 \text { g.cm }^{-3}\end{array}$ & Bulk water \\
& \multicolumn{2}{|c}{$\begin{array}{c}\text { [31] } \\
\mathrm{O}_{Z} \rightarrow \mathrm{O}_{W}\end{array}$} & $\mathrm{O}_{W} \rightarrow \mathrm{O}_{W}$ & $\mathrm{O}_{Z} \rightarrow \mathrm{O}_{W}$ & $\mathrm{O}_{W} \rightarrow \mathrm{O}_{W}$ \\
\hline Jump type & $\mathrm{O}_{W} \rightarrow \mathrm{O}_{W}$ & $\mathrm{O}_{Z}$ & $5.5 \pm 0.1$ & $2.5 \pm 0.1$ \\
$\tau_{\text {rror }}^{\mathrm{MD}}(\mathrm{ps})$ & $5.2 \pm 0.1$ & $4.8 \pm 0.1$ & $7.3 \pm 0.1$ & $\infty$ & $5.4 \pm 0.1$ \\
$\tau_{\text {reor }}^{\text {frame }}(\mathrm{ps})$ & $14.2 \pm 0.1$ & $\infty$ & $29.1 \pm 0.2$ & $35 \pm 1$ & $27 \pm 1$ \\
Libration cone angle $\left(^{\circ}\right)$ & $27 \pm 1$ & $40 \pm 1$ & $25 \pm 1$ & $74 \pm 2$ & $68 \pm 2$ \\
Jump angle $\Delta \theta\left(^{\circ}\right)$ & $58 \pm 2$ & $103 \pm 2$ & $60 \pm 2$ & $5.5 \pm 0.2$ & $3.3 \pm 0.1$ \\
Jump time $\tau^{\text {jump }}(\mathrm{ps})$ & $6.9 \pm 0.2$ & $5.5 \pm 0.2$ & $7.6 \pm 0.3$ & 5.5 & 3.6 \\
Jump reor. time $\tau_{\text {reor }}^{\text {jump }}(\mathrm{ps})$ & 9.0 & 4.4 & 9.5 & $5.5 \pm 0.3$ & $2.2 \pm 0.1$
\end{tabular}

Table 1: Characteristic times for water reorientation and the H-bond exchange process extracted directly from simulation and calculated via the extended jump model (EJM) ${ }^{[31]}$, as defined in the text. The librational cone angles are defined based on the reorientation time-correlation value after 200 fs using the procedure detailed in Ref. ${ }^{[32]}$.

\section{References}

[1] P. Ball, Chem Rev 2008, 108, 74-108.

[2] S. Bernèche, B. Roux, Nature 2001, 414, 73-77.

[3] N. Malikova, A. Cadène, V. Marry, E. Dubois, P. Turq, J Phys Chem B 2006, 110, 3206-3214.

[4] M. C. Bellissent-Funel, Eur Phys J E Soft Matter 2003, 12, 83-92.

[5] A. A. Milischuk, B. M. Ladanyi, J Chem Phys 2011, 135, 174709.

[6] D. Laage, W. H. Thompson, J Chem Phys 2012, 136, 044513.

[7] D. E. Moilanen, I. R. Piletic, M. D. Fayer, J Phys Chem C 2007, 111, 8884-8891.

[8] J. C. Rasaiah, S. Garde, G. Hummer, Annu Rev Phys Chem 2008, 59, 713-740.

[9] S. Paranthaman, F. X. Coudert, A. H. Fuchs, Phys. Chem. Chem. Phys. 2010, 12, 8124-8130.

[10] M. De Toni, R. Jonchiere, P. Pullumbi, F. X. Coudert, A. H. Fuchs, ChemPhysChem 2012, 13, 3497-3503.

[11] M. R. Harpham, B. M. Ladanyi, N. E. Levinger, K. W. Herwig, J Chem Phys 2004, 121, 7855-7868.

[12] I. R. Piletic, H. S. Tan, M. D. Fayer, J Phys Chem B 2005, 109, 21273-21284.

[13] M. D. Fayer, N. E. Levinger, Annu Rev Anal Chem 2010, 3, 89-107.

[14] D. E. Rosenfeld, C. A. Schmuttenmaer, J Phys Chem B 2011, 115, 1021-31.

[15] R. Costard, N. E. Levinger, E. T. J. Nibbering, T. Elsaesser, J Phys Chem B 2012, 116, 5752-5759.

[16] N. Giovambattista, P. J. Rossky, P. G. Debenedetti, Annu Rev Phys Chem 2012, 63, 179-200.

[17] E. E. Fenn, D. B. Wong, M. D. Fayer, Proc Natl Acad Sci USA 2009, 106, 15243-15248.

[18] S. M. Auerbach, K. A. Carrado, P. K. Dutta, Handbook of Zeolite Science and Technology, Marcel Dekker, New York, 2003.

[19] A. Corma, F. Rey, J. Rius, M. J. Sabater, S. Valencia, Nature 2004, 431, 287-290.

[20] P. Caullet, J. L. Paillaud, A. Simon-Masseron, M. Soulard, J. Patarin, C. R. Chimie 2005, 8, 245-266. 
[21] F. Cailliez, G. Stirnemann, A. Boutin, I. Demachy, A. H. Fuchs, J Phys Chem C 2008, 112, 10435-10445.

[22] F. X. Coudert, F. Cailliez, R. Vuilleumier, A. H. Fuchs, A. Boutin, Faraday Discuss 2009, 141, 377-398.

[23] D. Laage, G. Stirnemann, F. Sterpone, R. Rey, J. T. Hynes, Annu Rev Phys Chem 2011, 62, 395-416.

[24] F. X. Coudert, R. Vuilleumier, A. Boutin, Chemphyschem 2006, 7, 2464-2467.

[25] M. L. Connolly, J Am Chem Soc 1985, 107, 1118-1124.

[26] N. Desbiens, A. Boutin, I. Demachy, J Phys Chem B 2005, 109, 24071-24076.

[27] F. Cailliez, N. Desbiens, A. Boutin, I. Demachy, M. Trzpit, M. Soulard, J. Patarin, A. H. Fuchs, Stud. Surf. Sci. Catal. 2008, 174, $683-688$.

[28] N. Desbiens, I. Demachy, A. H. Fuchs, H. Kirsch-Rodeschini, M. Soulard, J. Patarin, Angew Chem 2005, 117, 5444-5447.

[29] F. X. Coudert, A. Boutin, Chem Phys Lett 2006, 428, 68 - 72.

[30] H. J. C. Berendsen, J. R. Grigera, T. P. Straatsma, J. Phys. Chem. 1987, 91, 6269-6271.

[31] D. Laage, J. T. Hynes, J Phys Chem B 2008, 112, 14230-14242.

[32] D. Laage, J. T. Hynes, Chem Phys Lett 2006, 433, 80-85.

[33] G. Stirnemann, P. J. Rossky, J. T. Hynes, D. Laage, Faraday Discuss 2010, 146, 263-281.

[34] G. Stirnemann, S. R.-V. Castrillón, J. T. Hynes, P. J. Rossky, P. G. Debenedetti, D. Laage, Phys Chem Chem Phys 2011, 13, 19911-19917.

[35] L. F. Scatena, M. G. Brown, G. L. Richmond, Science 2001, 292, 908-912.

[36] D. Laage, J. T. Hynes, Science 2006, 311, 832-835.

[37] D. Laage, G. Stirnemann, F. Sterpone, J. T. Hynes, Acc Chem Res 2012, 45, 53-62.

[38] F. Sterpone, G. Stirnemann, D. Laage, J Am Chem Soc 2012, 134, 4116-4119.

[39] R. Chanajaree, P. Bopp, S. Fritzsche, J. Kärger, Micropor Mesopor Mat 2011, 146, 106-118.

[40] D. Laage, G. Stirnemann, J. T. Hynes, J Phys Chem B 2009, 113, 2428-2435. 


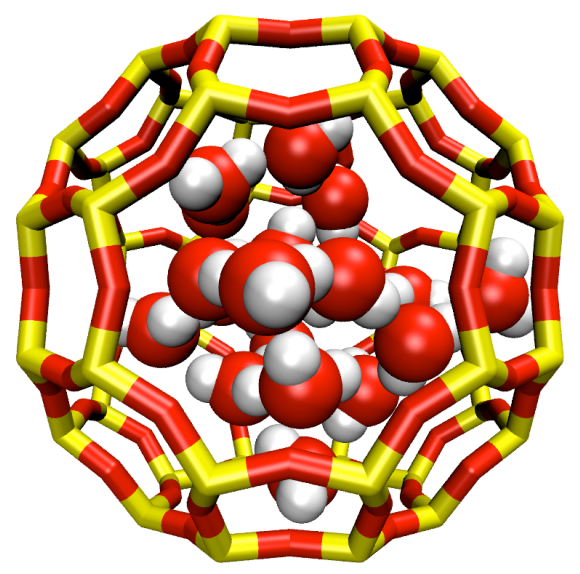

Figure 7: Table of contents. Water dynamics in extreme confinement: the presence of the interface has a much greater impact on reorientational dynamics than the additional structuring of water due to confinement. 